\title{
The Application of Ultrasonic Methods to Flow Measurement and Detection of Microembolus in Heart Prostheses
}

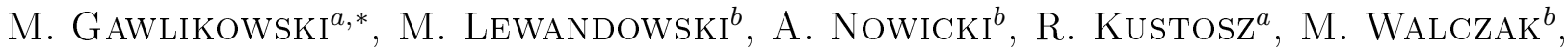 \\ P. KARWAT ${ }^{b}$ AND P. KAREOWICZ ${ }^{c}$ \\ ${ }^{a}$ Foundation of Cardiac Surgery Development, Wolności 345a, 41-800 Zabrze, Poland \\ ${ }^{b}$ Institute of Fundamental Technological Researches, PAS, Pawińskiego 5B, 02-106 Warsaw, Poland \\ ${ }^{c}$ Sonomed Ltd., Pruszkowska 4d, 02-118 Warsaw, Poland \\ For the last 20 years the world cardiosurgery has presented a considerable change of attitude to mechanical cir- \\ culatory support. In spite of technological progress the main problems in ventricular assist devices are: thrombosis \\ and low accuracy of flow measurements. In this paper the prototype of multi-gate Doppler flowmeter intended for \\ cardiac assist system ReligaHeart EXT has been presented as well as the possibility of ultrasonic microembolus \\ detection.
}

DOI: $10.12693 /$ APhysPolA.124.417

PACS: 43.35.Yb, 43.80.Qf, 43.35.Ae

\section{Introduction}

Latterly the mechanical circulatory support (MCS) has became clinical routine of heart insufficiency treatment [1-3]. Since the ' 80 s of the 20th century it has been conducted by means of pulsating, extracorporeal ventricular assist devices (VAD). Parallel to them the rotary blood pumps (RBP) were being developing. In spite of technological progress the MCS is inseparably connected with risks of blood traumatization (by contact with artificial material and mechanical stress [4]), bleeding and infection. The blood traumatization leads to decomposition of red blood cells (hemolysis) and activation of coagulation system (thrombogenicity), involving microembolus and well-organized clots growth, adhered to the internal surface of the blood pump. In spite of advanced methods of coagulation system blocking the thrombus remain principal side-effects of MCS [5].

In both types of VADs (pulsatile and RBP) the flow is not constant, therefore the ultrasound transit-time method is the most often used for its measurement [6] (other non-invasive methods [7-11] are in early stage of development). The accuracy of this assessment is about $5-7 \%$ for laminar flow [6] and less than $20 \%$ for transition or turbulent flow [12]. Due to the risk of clots forming the alternative method of measurements must be noninvasive. There is the only one clinically applied RBP including direct flow measurement [13], in other cases the flow is estimated based on electric power, speed and viscosity of blood $[14,15]$. This approach is low accurate $(20 \%$ for flows $>5 \mathrm{l} / \mathrm{min}[16])$ but the method itself is non-invasive and it allows to demonstrate the flow vs. time dependence.

The considerable achievements in the area of MCS by extracorporeal, pulsating VADs belong to Foundation of Cardiac Surgery Development [2]. Up till now more than

\footnotetext{
* corresponding author; e-mail: maciej.gawlikowski@poczta.onet.pl
}

270 application of POLVAD-MEV blood pumps have been performed. Based on the clinical experiences the Foundation has developed a new generation, extracorporeal cardiac support system ReligaHeart EXT [11, 17]. One of issues of the project concerned the development of non-invasive measurement methods intended for heart prostheses.

\section{Goal}

Despite technical progress the most particular problems concerned the contemporary MCS are thrombogenicity of VAD and blood flow measurement. In this paper authors would like to present:

- the results of investigations on the flow measurement by means of multi-gate Doppler,

- the perspective of microembolus detection by means of ultrasonic methods.

\section{Flow measurement}

It has been assumed that both pulsating and continuous volumetric flows should be measured by means of the Doppler method in the range of $\pm 5 \mathrm{l} / \mathrm{min}$ with accuracy about $10 \%$. The flow probe have to be mounted into the stub-pipe of the VAD made of biocompatible polyesterbased polyurethane.

\subsection{Material and methods}

The prototype of the flowmeter has been developed based on the multi-gate pulse wave Doppler technique [18], in which the volumetric flow is calculated by integration of the flow velocities at each gate. The system consists of two modules: the first is responsible for emitting, receiving and processing of the $\mathrm{RF}$ signal, the second (a digital one) demodulates, filters and post-processes the signal. The received ultrasound echo is amplified and digitalized. The next step of the processing is the digital down conversion (DDC) and filtration by means of the cascaded-integration-comb filter (CIC) implemented on the FPGA. The DDC block consists of two multipliers, reference signal generator $(1-16 \mathrm{MHz})$ and two low-pass filters. The samples of the echo are mixed with 14-bit samples of sine and cosine reference signals. As a result two signals: in-phase $(I)$ and quadrature $(Q)$ are produced. Subsequently, both $I$ and $Q$ signals are filtered 
by a low-pass filter and decimated to obtain the baseband signal. In multi-gate system, the signal in each gate has to be filtered in order to remove the low-frequency and high-amplitude components that are induced by static objects (echoes). In the FPGA 200 parallel finite impulse response filters have been implemented. These allow to elaborate 100 Doppler gates in real-time. Then, the signal is transmitted to the PC for further post-processing.

To develop the probe the sound velocity and attenuation should be assessed. Three samples made of polyurethane have been measured. Assuming the ultrasound frequency of $4 \mathrm{MHz}$ and the length of beam propagation about $5 \mathrm{~mm}$ the calculated average suppression was $0.61 \mathrm{~dB} /(\mathrm{mm} \mathrm{MHz})$ and the sound velocity was $1880 \mathrm{~m} / \mathrm{s}$. The angle between transducer and surface of the connector was calculated according to the Snellius' law. The Doppler angle of $60^{\circ}$ (routine for medical application [19]) was assumed. A direction of the ultrasound beam penetration into blood was investigated by means of the Schlieren method (Fig. 1).

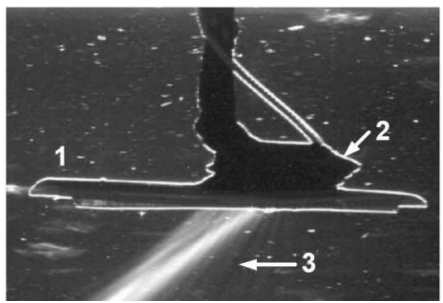

Fig. 1. The visualization of the ultrasound beam by means of the Schlieren method.

Selection of an ultrasound frequency was made as a compromise between wave attenuation, spatial resolution and maximum flow velocity. Taking into consideration the geometry of the connector, the maximum depth of measurements is less than $40 \mathrm{~mm}$, thus the pulse repetition frequency was set to $16 \mathrm{kHz}$. At the frequency of $4 \mathrm{MHz}$ the maximum measurable speed of flow is higher than $3 \mathrm{~m} / \mathrm{s}$.

Due to further application a transducer diameter should be as small as possible. On the other hand, a range of the near field and lateral size of the beam should be taken into consideration. The optimal range of distances from the transducer for $-6 \mathrm{~dB}$ criterion and various diameters is shown in Table. In a prototype of flowmeter the transducer with a diameter of $5 \mathrm{~mm}$ was applied.

TABLE

Regions of beam focusing vs. diameter of the transducer.

\begin{tabular}{c|c|c|c|c|c}
\hline \hline Diameter [mm] & 3.0 & 3.5 & 4.0 & 5.0 & 6.0 \\
\hline beginning [mm] & 3.8 & 5.2 & 6.8 & 10.6 & 15.3 \\
end [mm] & 11.5 & 15.6 & 20.4 & 31.8 & 45.8
\end{tabular}

The demodulated $I / Q$ signal is processed by means of a complex Fourier transform. The Doppler spectrum is represented as a function of time $t$ and depth $g$ (the gate number). The noisy background is removed by means of an adaptive cut-off and for each spectrum an average frequency is calculated according to the equation (1). Thus, the map of average Doppler frequencies is carried out.

$$
F_{\mathrm{AV}}(g, t)=\int f S(f, g, t) \mathrm{d} f / \int S(f, g, t) \mathrm{d} f .
$$

The map of $F_{A V}$ is converted to the velocity $v$ of the flow at the gate $g$ by means of the equation

$$
v(g, t)=\int F_{\mathrm{AV}}(g, t) \frac{c}{2} / \int F_{n} \cos \alpha
$$

where $F_{n}$ - frequency of emitted signal, $c$ - sound velocity in blood, $\alpha$ - angle between ultrasound beam and direction of the flow. The instantaneous volumetric flow $Q(t)$ is calculated as a sum of fluxes corresponding to the velocities of the flow $v(g, t)$ at respective gates $g$

$$
Q(t)=\sum v(g, t) \mathrm{d} S(g),
$$

where $\mathrm{d} S(g)$ - the part of a vessel lumen assigned to the gate.

The tests of developed system consisted of comparison of volumetric flow (continuous up to $7 \mathrm{l} / \mathrm{min}$ and pulsating up to $5 \mathrm{l} / \mathrm{min}$ ) with the reference (the transit-time flowmeter T-206/Transonic, uncertainty about $8 \%$ ). The flow was generated using POLVAD-MEV (FRK) and BP80 (Medtronic) blood pumps, respectively. The operation liquid was porcine blood anticoagulated by means of CPDA-1. For the purpose of experiment a model of connector was developed by means of a rapid prototyping. The model allowed to apply six transducers at different spatial arrangement. In order to find the highest accuracy the flow measurements were carried out for all transducers.

\subsection{Results}

The results of measurements of pulsating $\left(Q_{A V}=\right.$ $3 \mathrm{l} / \mathrm{min}$ ) flows have been presented in Fig. 2. The gray scale represents power of the reflected ultrasound signal and mean Doppler frequencies, respectively. The volumetric flow vs. time was calculated according to the Eq. (3). The accuracy of flow measured by multi-gate Doppler has been presented in Fig. 3.

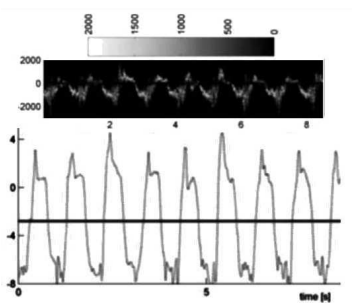

Fig. 2. Results of flow measurement by means of multigate Doppler (for pulsating flow).

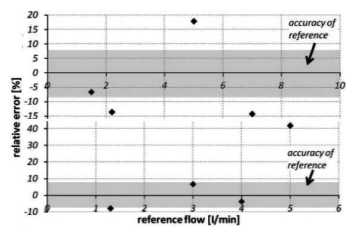

Fig. 3. Accuracy of flow measurements by means of multi-gate Doppler. 


\subsection{Discussion}

In the range of $4 \mathrm{l} / \mathrm{min}$ the accuracy of pulsating flows measurements is comparable to the reference (Fig. 3 ) but the accuracy of continuous flow measurements is about two times worse than the reference and reaches $15 \%$. Because the volumetric flow is calculated based on the local flow velocities it is presumed, that observed effect is connected with high variance of local flow caused by: its turbulent character during flow through the diffusershaped connector or the detachment of the stream [20]. Due to inertia of blood the dynamics phenomena occurring in pulsating flow cannot be considered as a simple combination of excerpts of continuous one.

In order to assess the flow instability the FEM simulations may be utilized, however (due to complicated modeling of blood rheology [21]) the results ought to be verified in physical experiment, e.g. by means of ultrasonic Doppler velocimetry [22]. In this work such measurements (commercial apparatus DOP2000/SignalProcessing, probe $4 \mathrm{MHz}$ ) has been carried out for continuous flow $(4 \mathrm{l} / \mathrm{min})$ at the outlet connector and $10 \mathrm{~cm}$ behind it, at the rectilinear tube. The analysis of velocity profiles (average AV and standard deviation $\mathrm{STD}, n=200$, sampling time $100 \mathrm{~ms}$ - refer to Fig. 4) confirmed that the flow character at connector is much more turbulent than at the cannula (non-parabolic shape of AV curves, Reynolds' number about 11000 but the variance of flow through the cannula is 2.5 times lower than through the connector).

It should be noticed that the investigated multi-gate Doppler system was able to measure pulsating flows with sufficient dynamics for application in VAD. It is possible to assess the static and dynamic backflows through the mechanical valves and to detect the malfunction of the valve caused by clots or mechanical defect.

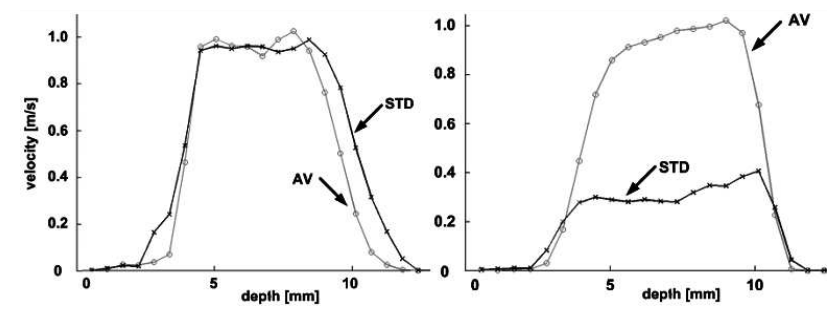

Fig. 4. Velocity profiles of flow measured at connector (left side) and rectilinear tube (right side).

\subsection{Conclusions}

Developed multi-gate Doppler system allows to measure average flow as well as pulsating with high dynamics. For average flows less or equal to $4 \mathrm{l} / \mathrm{min}$ the accuracy is comparable with the reference (about $8 \%$ ). For flows greater than $5 \mathrm{l} / \mathrm{min}$ the accuracy decreases significantly. The small-diameter $(5 \mathrm{~mm})$ transducers may be mounted into the connector of the VAD. Further development of the system should focus on assuring adequate accuracy within whole range of measured flows.

\section{Possibility of microembolus detection} by means of Doppler methods

The reflected ultrasound echo contains surplus information, e.g. its power is connected with the acoustic features and size of reflecting objects. It may be utilized to detection of microclots or to monitoring the air bubbles in MCS systems. The Doppler ultrasound is a convenient tool for monitoring of embolization in the cerebral circulation. It is widely used to detect emboli, resulting from artificial heart valves and several clinical procedures, such as carotid artery surgery or cardiac surgery. The typical signal of numerous microclots registered in left carotid artery has been shown in Fig. 5 [23] (distinctive light stripes correspond to high power of echo). It should be noticed, that the same effect occurred during blood circulation in mock loop ( Fig. 2, the Doppler spectrogram). A standard approach involves human experts who distinguish embolic events from background and artifacts, however, many papers introduce systems for automated emboli detection [24, 25].

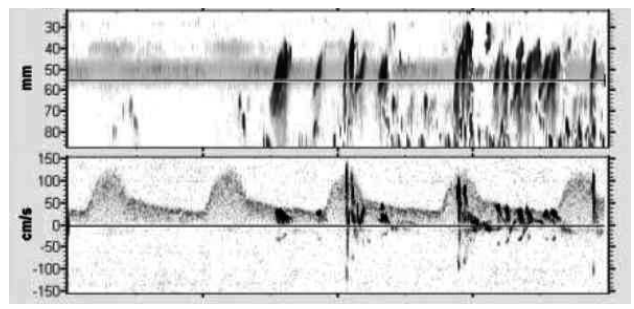

Fig. 5. Ultrasonic signal characteristic for numerous microembolus (measurements carried out in right and left carotid artery during cardiac catheterization) [25].

As the embolic signal is highly non-stationary, researchers use and compare various kinds of bilinear timefrequency representations of the data [26]. Some of them report on the advantage of the wavelet transform over the short-time Fourier transform [27]. Moreover, the emboli detection methods are being improved through new signal representation techniques which take advantage of the data from a range of depths rather than from a single gate (e.g. the Power M-mode Doppler technique [28]). Other paper [29] presents a principal component analysis of the RF data in the time-depth domain. Above techniques improve the sensitivity of the emboli detection methods and allow for removal of the human factor. However, a composition and a size of the detected emboli remain unknown. In terms of the amplitude of echoes, small bubbles behave similarly to larger solid emboli. Thus, the analysis of the reflected power is insufficient to determine the matter of the emboli or to estimate their size. To overcome this limitation more sophisticated techniques are required.

One method [30, 31] allows to distinguish the gaseous emboli from the solid ones by taking advantage of their non-linear behavior. The air bubbles not only reflect the fundamental frequency but also convert some of the acoustic energy into harmonic frequency components. As the diameter of the gaseous emboli determines their reso- 
nance frequency, it can be estimated with this technique as well. This method, however, requires special transducer design which allows to transmit adequate scanning pulses and at the same time provides wide receiver band to acquire the frequency components. Moreover, it still doesn't provide the information on the size of the solid emboli.

In order to characterize both the gaseous and solid emboli, an emboli-to-blood ratio (EBR) was introduced [32]. However, this parameter is based on backscatter cross-section of emboli and blood flowing through the sample volume, and thus it cannot be measured directly. Therefore, practical implementation of this idea involves a measured EBR (MEBR) which is defined as a ratio of powers of signals from a sample volume in the presence and absence of embolic events [33]. Fortunately, the MEBR to embolus diameter dependence varies with frequency, and thus use of more than one transmitted frequency potentially allows to overcome the mentioned ambiguity in determination of the emboli size.

Knowledge in the area of multi-gate Doppler flow measurements and development of extracorporeal heart prosthesis ReligaHeart EXT have formed the basis of research project called "clotALERT", realized by Foundation of Cardiac Surgery Development and Institute of Fundamental Technological Researches. Goals of the project are: to improve the ultrasonic multi-gate Doppler flowmeter in order to increase the accuracy and to develop the methods and system of ultrasonic detection of microembolus, capable to estimate its number and diversify its size. The in-vitro experiments will be carried out on modified construction of ReligaHeart EXT blood pump and on porcine blood.

\section{Acknowledgments}

The multi-gate Doppler system has been developed as a part of the Polish Artificial Heart Program. The "clotALERT" project is supported by The National Centre of Research and Development, PBS1/A3/11/2012.

\section{References}

[1] J. Garbade, H.B. Bittner, M.J. Barten, F.W. Mohr, Cardiol. Res. Practice 2011, 290561 (2011).

[2] J. Pacholewicz, E. Kucewicz, M. Zakliczyński, T. Niklewski, K. Kubacki, R. Kustosz, M. Zembala, Kardioch. Torakoch. Pol. 5, 308 (2008) (in Polish).

[3] P. Litwiński, B. Woźniewicz, G. Religa, M. Pastuszek, A. Parulski, M. Jasińska, Sz. Kocańda, R. Kustosz, P. Siondalski, Z. Religa, Kardiochir. Torakochir. Pol. 2, 33 (2005).

[4] M.H. Kroll, J.D. Hellums, L.V. McIntire, A.I. Schafer, J.K. Moake, Blood 88, 1525 (1996).

[5] S.R. Wilson, M.M. Givertz, G.C. Stewart, G.H. Mudge, J. Am. Coll. Cardiol. 54, 1647 (2009).

[6] G. Beldi, A. Bosshard, O.M. Hess, U. Althaus, B.H. Walpoth, Ann. Thorac. Surg. 70, 212 (2000).

[7] D. Komorowski, M. Gawlikowski, Adv. Soft Comput. 45, 558 (2007).

[8] T. Pustelny, J. Ignac-Nowicka, Z. Opilski, Opt. Appl. 34, 249 (2004).
[9] Z. Opilski, G. Konieczny, T. Pustelny, A. Gacek, R. Kustosz, M. Gawlikowski, Bull. Pol. Acad. Sci. Tech. Sci. 59, 429 (2011).

[10] A. Sobotnicki, T. Pałko, J. Mocha, M. Czerw, J. Med. Inform. Technol. 19, 117 (2012).

[11] G. Konieczny, Z. Opilski, T. Pustelny, M. Gawlikowski, Biomed. Eng. Online 11, 72 (2012).

[12] L.C. Lynnworth, Y. Liu, Ultrasonics 44, e1378 (2006).

[13] E.V. Potapov, A. Koster, M. Loebe, E. Hennig, T. Fischer, R. Sodian, R. Hetzer, J. Extra Corpor. Technol. 35, 274 (2003).

[14] N. Malagutti, D.M. Karantonis, S.L. Cloherty, P.J. Ayre, D.G. Mason, R.F. Salamonsen, N.H. Lovell, Artif. Organs 31, 45 (2007).

[15] M. Granegger, F. Moscato, F. Casas, G. Wieselthaler, H. Schima, Artif. Organs 36, 691 (2012).

[16] HeartWare Ventricular Assist System - Instruction for use. Rev5/02/09. HeartWare Inc., Miami 2009.

[17] M. Gawlikowski, R. Kustosz, A. Kapis, W. Bujok, Z. Małota, K. Janiczak, L. Czerwiński, M. Czak, M. Głowacki, M. Gonsior, "The New Generation of Cardiac Assist System Religa Heart EXT", Adv. Biomed. Eng., in press (in Polish).

[18] W. Secomski, A. Nowicki, P. Tortoli, R. Olszewski, Ultrasound Med. Biol. 35, 230 (2009).

[19] J. Gao, K. Hentel, Q. Zhu, T. Ma, G. Shih, K. Mennitt, R. Min, Int. J. Nephrol. Renovasc. Dis. 4, 49 (2011).

[20] J. Li, Dynamics of the Vascular System, World Sci., Singapore 2004

[21] M. Gawlikowski, T. Pustelny, R. Kustosz, J. Phys. IV: JP (France) 137, 73 (2006).

[22] V. Koeseli, S. Zeybek, Y. Uludag, Turk J. Chem. 30, 297 (2006).

[23] M. Hamon, F. Burzotta, C. Oppenheim, R. Morello, F. Viader, Trials 8, 15 (2007).

[24] M.A. Moehring, M.P. Spencer, Ultrasound Med. Biol. 28, 49 (2002).

[25] L. Fan, D.H. Evans, A.R. Naylor, Ultrasound Med. Biol. 27, 1065 (2001).

[26] B.S. Krongold, A.M. Sayeed, M.A. Moehring, J.A. Ritcey, M.P. Spencer, D.L. Jones, IEEE T. Biomed. Eng. 46, 1081 (1999).

[27] P. Palanchon, A. Bouakaz, J. Klein, N. De Jong, Ultrasound Med. Biol. 29, 417 (2003).

[28] L. Fan, E. Boni, P. Tortoli, D.H. Evans, "Development of a Dual-Gate Automatic Embolus Detection System", in: 2004 IEEE Ultrasonics Symp. 2004.

[29] M.A. Moehring, J.R. Klepper, IEEE T Bio-Med. Eng. 41, 35 (1994).

[30] E. Roy, P. Abraham, S. Montresor, J.-L. Saumet, Ultrasound Med. Biol. 26, 419 (2000).

[31] P. Palanchon, A. Bouakaz, J. Klein, N. De Jong, Ultrasound Med. Biol. 30, 123 (2004).

[32] D.H. Evans, ASUM Ultrasound Bull. 10, 19 (2007).

[33] J. Cowe, D.H. Evans, Ultrasound Med. Biol. 32, 1853 (2006). 